\title{
¿Espacios sociales con economías solidarias? El caso del Movimiento de los Sin Techo Metropolitano y del Perú
}

Recibido: 04/10/2016

Aprobado: 15/11/2016

\author{
Daniel Fonseca Zanca, Kelly Jara Gómez, \\ Giancarlos Espinoza Malpartida, \\ Fredy Chirinos Quispe, \\ Julio Méndez Leguía \\ Universidad Nacional Mayor de San Marcos
}

\begin{abstract}
RESUMEN
El trabajo aborda la existencia de relaciones de economía social y solidaria dentro del Movimiento de los Sin Techo Metropolitano y del Perú (MST-MP). Este movimiento surge por el derecho a la vivienda en la ciudad de Lima y en el marco del largo proceso histórico de tomas de tierra producido como parte de la urbanización de zonas periféricas de diversas ciudades del país. El trabajo analiza cuatro ejes principales: historia y memoria de la experiencia, organización y conflictos internos, relación con actores de su entorno y las perspectivas de futuro.
\end{abstract}

Palabras clave: Economía social y solidaria, ciudad, tomas de tierra, movimientos sociales urbanos.

\section{Spaces of social with solidarity economy? The case of the Movement of the homeless Metropolitan and of Peru}

\begin{abstract}
The paper deals with the existence of relations of social and solidarity economy within the Movement of the Homeless Metropolitan and Peru (MST-MP). This movement arises from the right to housing in the city of Lima and within the long historical process of land occupations occurred as part of the development of peripheral areas of various cities. The work analyzes four main themes: history and memory of the experience, organization and internal conflicts, relationship with actors of their environment and future prospects.
\end{abstract}

KeYworDs: Social and solidarity economy, city, land occupations, urban social movements. 


\section{Introducción}

"No hay soluciones fáciles y cualquiera de ellas va a exigir compromiso, organización y movilización social. La condición fundamental para que las y los de abajo influyan y tengan voz es crear un auténtico poder ciudadano. Hay que indignarse, rebelarse $y$, sobre todo, luchar."

Manuel Monereo, "Por la revolución ciudadana", El viejo topo,

20 de mayo de 2011

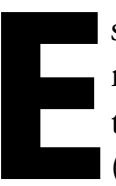

ste trabajo analiza si existen relaciones de economía social y solidaria dentro del Movimiento de los Sin Techo Metropolitano y del Perú (en adelante MST-MP), que surge en la ciudad de Lima en el año 2011, con la reivindicación programática central de luchar por el derecho a la vivienda. No pretende esclarecer si esta organización tiene el carácter de "movimiento" o busca contrastar su experiencia con las teorías dedicadas a los movimientos sociales, sino más bien, estudia su dinámica como organización económico social.

La investigación está orientada al estudio de formas alternativas a la economía capitalista, centrándose en los aspectos de su organización de recursos y los valores propios de este tipo de economía o economías, así como su relación con la urbe. Teniendo como base, las teorías sobre las relaciones de economía social y solidaria ${ }^{1}$, pretendemos verificar de qué forma estas se presentan en el proceso de construcción de esta organización económico social y observar qué otro tipo de economía puede ser desenvuelta, dentro del contexto de la lucha por la vivienda y las tomas de tierras desenvueltas en una ciudad como Lima.

El trabajo aborda cuatro asuntos: Primero, el fenómeno de surgimiento del MST-MP, el cual constituye un suceso nuevo en la historia del ciclo largo de los movimientos urbanos del Perú2. Por ello, recupera la memoria de este movimiento para profundizar en las circunstancias que permitieron su conformación y el

$1 \quad$ La economía social y solidaria según la propuesta de Coraggio (2011) esta orientada al "desarrollo y equidad, democratización de la economía, consumo responsable, comercio justo, justicia social, solidaridad, reciprocidad, reproducción ampliada de la vida, Buen Vivir, Vivir Bien; pero todo esto como producto de una construcción colectiva, social y política en el campo de las fuerzas, producto de la relación entre trabajo y capital.

2 La historia de los movimientos urbanos en el Perú del siglo XX fue analizada con detalle por Meneses (1998). El MST-MP surge en un contexto socio-histórico posterior y donde la ciudad de Lima despliega nuevas dinámicas. por qué fue necesario su surgimiento, dando alcances del contexto socio-histórico y su expansión territorial como organización. Segundo, igualmente es determinante el análisis de las relaciones internas de la organización, particularmente, de sus conflictos. Tercero, analiza los vínculos que establece con los diversos actores y agentes con los cuales establece negociaciones, acuerdos y tensiones. Cuarto y final, indaga en las perspectivas de futuro de esta organización, en lo referido al curso de las relaciones económico sociales y solidarias que desenvuelve y sus implicancias en la lucha por el derecho a la vivienda.

Este trabajo asume una metodología de investigación acción y utiliza herramientas de recojo de información de tipo cualitativo, como la observación participante y entrevistas a profundidad, aplicadas a personajes representativos de esta organización, como dirigentes y activistas, varones y mujeres.

Agradecemos a los compañeros y compañeras del MST-MP por la disposición y la ayuda brindada a nuestro grupo de investigación, por permitirnos el acceso y contacto con los lugares y personas que brindaron la información usada en este trabajo. Agradecemos también al profesor Luis Montoya Canchis por animarnos a realizar esta experiencia de indagación.

\section{Recuperación de memoria}

\section{a. Antecedentes}

A partir de la década del cincuenta, el crecimiento demográfico de Lima fue acelerado con las olas migratorias del campo a la ciudad. Tal fue el efecto que Lima se expandió territorialmente formándose los denominados "conos", y posteriormente, recibieron el nombre de "Limas", norte, este y sur.

La existencia de una población significativa, que al no contar con ingresos que les permitieran cubrir los precios de suelo y vivienda, determinó el inicio de diversos procesos de ocupación o tomas de tierra, de amplias y diversas áreas de la ciudad de Lima.

La historia de este proceso nos muestra una figura simbólica ineludible de mención, porque sintetiza en gran medida esta larga y compleja trayectoria: Ernesto Sánchez Silva "Poncho negro". Él fue quien se convirtió en el padre de las tomas de tierra. Tras la primera gran movilización, organizada en 1964, que ocupó el cerro 7 de octubre, convirtió este hecho en 
un oficio que sumó a sus dotes de artista, cantante y maestro cocinero. Durante tres décadas, "poncho negro" organizó y dirigió al menos 132 tomas de tierra de principio a fin, teniendo como consigna: "Las tierras no son de nadie, son de Dios y para sus hijos" (García, 2013).

Así nacieron muchos pueblos jóvenes que en algunos casos se convirtieron en urbanizaciones, como en el caso de los distritos de El Agustino, Comas, San Juan de Lurigancho y otros de Lima, produciéndose constantes luchas y enfrentamientos por el derecho a la vivienda. Arroyo y Romero (2008) señalan al respecto:

De esta manera, se fueron configurando las nuevas centralidades en los grandes espacios que hasta los años 1980 constituían la periferia de la expansión urbana, conocida como "el cinturón de miseria" y ahora como los "conos" de Lima. De ser asentamientos dormitorios pasaron a alojar una población precaria e informal que generará una base económica desconcentrada, gracias a su dinamismo socio-demográfico y a la creciente concentración/aglomeración de micro y pequeńas unidades principalmente dedicadas al comercio, junto con otras de producción artesanal y manufacturera. En términos de densidad demográfica y dinamismo económico, las nuevas ocupaciones fueron ganando peso y notoriedad durante las dos últimas décadas del siglo XX. (2008:107).

Sin embargo, las y los desposeídos, aquellas y aquellos que no contaban con un techo o vivienda, continuaron protagonizando tomas de tierra. Un sector de ellas y ellas protagoniza en el 2010, en el sur de la ciudad de Lima, una gran movilización, motivada por una toma de tierras fallida en la zona arqueológica de Lurín, donde convocaron a conseguir un terreno a costos bajos. La movilización origina una marcha con cerca de 10,000 familias, vestidas con polos blancos, y encabezada por dirigentes y dirigentas cubiertos con pochos del mismo color, en conmemoración a la figura emblemática de Ernesto Sánchez Silva "poncho negro"

Esta movilización es uno de los hitos fundacionales del MST-MP, porque representa una continuidad con las tomas de tierra producidas en décadas anteriores y con un profundo valor simbólico para sus protagonistas, no sólo reflejado en el uso ritual de los pochos blancos, sino porque nacía de una necesidad sentida para ellas y ellos: La lucha por el derecho a la vivienda.

$3 \quad$ Versión brindada por Rocío Medina dirigenta del MST-MP de la base de San Juan de Miraflores.

\section{b. Movimiento de los Sin Techo Metropolitano y del Perú}

El MST-MP, es fundado el 12 de junio de 2011, con la reivindicación programática principal de conseguir una "vivienda digna" para las familias que no cuentan con esta a causa de la insuficiencia de políticas públicas que atendieran este derecho.

Esta organización fue fundada por dirigentes sociales con experiencia organizativa, que en un inicio habían tenido participación en tomas de tierras o en experiencias de organización con pobladores de zonas urbano populares.

El MST-MP no sólo va tener por objetivo la consecución de terrenos para las familias que lo necesitan, sino también procurará conseguir los servicios básicos que les servirán para lograr una "vivienda digna".

Las relaciones que serán la base del proceso organizativo de la experiencia del MST-MP estarán basadas en el voluntariado y diversas formas de trabajo no remunerado, porque sus dirigentes no cobrarán por su labor. Tampoco dependerá de algún agente externo, en términos financieros o de algún otro tipo de recurso; y por ello, tendrá una fuerte autonomía frente agencias del Estado, organizaciones no gubernamentales, partidos políticos, iglesias o empresas privadas.

Es necesario anotar que luego de la aprobación de su primer estatuto, los intereses individuales de varios dirigentes, generaron tensiones y fraccionamientos dentro de la organización que fueron sucediéndose y multiplicándose casi desde el inicio de su creación. La primera base afectada por estas tensiones y rupturas fue la base de Lima norte.

El primer presidente del MST- MP, José Pérez Juárez, mantuvo confrontaciones durante su gestión, y al no desempeñar eficientemente su cargo ni deslindar sus responsabilidades fue "dado de baja". Pérez Juárez, luego de su expulsión, seguió usando el nombre del MST, debido a que estaba inscrito en los Registros Públicos como dirigente. Él organizó además una facción que generó una serie de controversias y conflictos, tanto internos como externos.

La debilidad institucional es agudizada con la ruptura protagonizada por el presidente de la base del distrito de Independencia, William Cocha, que genera mayores divergencias y tensiones.

Esta serie de sucesos aceleraron un cambio del estatuto y a la vez determinaron la elección de una nueva directiva en el ańo 2012. El resultado es la designación de Rubén Pérez Flores como nuevo presidente. 
La relación con la gestión de la alcaldesa Susana Villarán, quien asume la conducción de la Municipalidad Metropolitana de Lima entre los años 2011 y 2014, constituye un momento clave de la historia del MST-MP, por las oportunidades que abrió para su movilización; pero también por las tensiones que provocó al no haber reconocido varias de sus reivindicaciones programáticas.

Es así que, en el transcurso del año 2012, el MSTMP establece lazos con la Municipalidad Metropolitana de Lima, sobre todo luego del inicio del proceso de revocatoria de autoridades municipales, tanto de la alcaldesa Villarán como de sus regidoras y regidores.

Durante el proceso de revocatoria el MST-MP apoyó a Susana Villarán, a fin de evitar su salida del gobierno municipal, a través de marchas masivas y mítines, que sucedieron en gran medida en el sur y norte de la ciudad.

En este proceso, la alcaldesa Villarán planteó establecer un pacto, que consistía en que una vez que el MST-MP apoyara su mantenimiento en el cargo y, por lo tanto, se lograra derrotar su intento de revocatoria, esta daría su apoyo a un proyecto de ordenanza sobre "vivienda digna", presentado por el MST-MP, para que fuera aprobado por el concejo de la Municipalidad Metropolitana de Lima.

En efecto, la alcaldesa Villarán consiguió mantenerse en su puesto y de otro lado, el MST-MP logró la promulgación de la ordenanza municipal $\mathrm{N}^{\circ} 1643-$ Programa Municipal de Vivienda Popular, el 22 de diciembre de 2012.

Pero la implementación de la norma fue congelada porque el presupuesto prometido para financiar el programa fue destinado hacia el programa "Barrio mío", una de las propuestas priorizadas por la alcaldesa Villarán durante la etapa final de su gestión.

Más adelante, con el apoyo de CENCA, y otras organizaciones no gubernamentales, consiguen mejorar la ordenanza y convertirla en un proyecto de ley gracias al soporte técnico legal y las capacitaciones permanentes que estas organizaciones les brindaron.

Sin embargo, para dar continuidad a la experiencia del MST-MP, se desenvolvieron otras iniciativas que fueron más allá del campo de la incidencia política. $\mathrm{Su}$ esfuerzo fue encaminado a conseguir terrenos para vivienda, en la medida que aumentaban los programas habitacionales fomentados desde el Estado; pero basando su propuesta en mecanismos autogestionarios y autofinanciados por las y los integrantes del MST-MP.
Por ello, en el año 2014, se implementa el Proyecto de Vivienda Jesús Nazareno, en la zona de Collique, del distrito de Comas. Su implementación representa un logro importante porque constituye una muestra concreta de la posibilidad del MST-MP de brindar viviendas a sus asociados. El impacto favorable generado por el proyecto dentro de la organización provoca que el 2 de agosto se reelija a Rubén Pérez Flores como presidente del MST- MP.

La experiencia del MST-MP es sui genis y muestra luego de su recorrido de cerca de cinco años un potencial aún por desenvolver. No obstante, a pesar que el MST-MP ha ido creciendo y expandiendo su presencia en diferentes distritos de la ciudad de Lima, aún no posee un núcleo de dirección fortalecido que una a sus diferentes bases y logre cohesionar su estructura organizativa interna; pero su experiencia parece soportarse en un modelo autogestionario y de fuerte preservación de su autonomía.

\section{Organización y conflictos Internos}

Esta sección esta dedicada a analizar la estructura organizacional y dinámica interna del MST-MP. Además de las principales tensiones que afronta como parte de su dinámica de funcionamiento.

\section{a. Organización interna}

Las secretarias y funciones dentro del MST-MP, son desempeñadas por un periodo de dos ańos, incluida la de la presidencia, las mismas que son elegidas de manera democrática y abierta. La composición y desempeńo de las funciones de cada uno de los miembros esta esquematizado de la siguiente manera:

Presidencia: Rubén Pérez.

Vicepresidencia: Susana Paz.

Secretaria de organizaciones: Martín Ypanaque.

Secretaria de actas: Estela Paucar.

Tesorería: Rocío Medina.

Vocal 1: Cesar Viera.

Vocal 2: Aparicio Rondón.

Secretario de asistencia social: Cesar Viera.

Secretaria de cultura y deporte: Deiver Veramendi.

Secretaria de disciplina: Jesús Lipa.

Secretaria de prensa y propaganda: Diego Mamani.

Una de las caractericas del MST-MP es estar integrado principalmente, en su mayoría, por dirigentes de 
Lima Norte y Lima Sur, siendo estas dos las principales zonas de la ciudad de Lima donde tiene presencia organizada.

Su accionar a nivel distrital esta concentrado principalmente en los distritos de Comas, Los Olivos e Independencia (Lima norte), Villa El Salvador, Villa María del Triunfo, San Juan de Miraflores, Chorrillos (Lima sur). Además ha intentado organizar bases en San Juan de Lurigancho (Lima este).

El trabajo de los dirigentes es fundamental, en sus respetivas bases, con sus asociados. Cada dirigente tiene la misión de activar y sumar más asociados, recoger sus demandas y necesidades y buscar los mecanismos necesarios para darle solución.

Podemos resaltar la equidad de género, entre varones y mujeres, en la participación desenvuelta en las secretarias, así como la presencia de cuadros jóvenes en el núcleo central de la organización.

El modelo organizativo del MST-MP es de carácter autogestionario, porque depende de los recursos autogenerados por sus asoaciados y relaciones sustentadas en la solidaridad, la cooperación y vínculos basados en la reciprocidad.

Señaladas estas características, pasaremos a analizar algunos de los principales conflictos que experimenta la organización.

\section{b. Conflictos}

El accionar de los miembros del MST-MP, pretende buscar terrenos privados y comprarlos, para la formación de programas de vivienda popular, es uno de los principales puntos de tensión en la organización, ya que la misma significa tomar una nueva actitud en cuanto a las acciones que desenvolvieron en épocas pasadas algunos de sus actuales dirigentes y que estuvieron centradas en la toma de tierras.

Este señalamiento nos lleva al análisis del caso actual, del primer programa de vivienda impulsado por el MST-MP, el Proyecto Especial de Vivienda Jesús Nazareno de Collique, Comas, el cual se convirtió en el máximo estandarte de la gestión de Rubén Pérez Flores, como presidente.

La tensión surge del hecho que el proyecto será visto por un sector de los dirigentes del MST-MP, en especial en el caso de los dirigentes de la base de Lima norte, como su máximo logro. y en algunos momentos su labor sólo se reducirá a dicha base, dejando de lado la problemática que afectaba al conjunto del movimiento en toda la ciudad de Lima.

Además la centralización de las reuniones en Lima norte, que a partir del proyecto serán realizadas en su gran mayoría en dicha base, y en muy pocas oportunidades en otras, generarán diferencias y malestar entre los asociados, principalmente, de Lima sur.

El proyecto no sólo genera tensiones internas. Evidencia también deficiencias. La principal es que carece de inscripción en registros públicos. La misma que aún no puede ser emitida, dado que el actual dueño del terreno no hace la debida inscripción, porque espera el pago completo de todos los asociados. Esto ha generado diversos cuestionamientos y acusaciones de malos manejos entre dirigentes. Además el retraso y la demora en el trámite de la habilitación urbana y la zonificación también han provocado diversas críticas y reclamos.

Este hecho sin duda, ha polarizado en algunos momentos a los miembros del movimiento, representando la tensión más evidente, el conflicto entre las y los dirigentes de las bases de Lima norte y Lima sur. Estos últimos cuestionan permanentemente el liderazgo de Rubén Pérez, criticando su estilo personalista y caudillista.

Esto se evidencia en un fraccionamiento de las bases, por un lado los de norte, con sus problemas; y por el otro, los del sur con sus demandas. Como que de alguna manera, se van aislando cada quien con su base. Lo que generó que en años anteriores, el movimiento sólo se quedara en buenas intenciones y no emprendiera nuevos retos.

Sin embargo, dichas tensiones evidenciadas y expuestas en sus respectivas reuniones, han sido escuchadas y por eso la proyección y nuevas acciones del movimiento ahora. En la secuencia de esta lógica de la búsqueda de nuevos terrenos, para la conformación de viviendas populares, ha surgido el proyecto de San Juan de Lurigancho ubicado al este de Lima. El cual será visto con mayor cuidado y rigurosidad en su realización. Los dirigentes comprendieron que no pueden repetir los mismos errores del primer proyecto de vivienda de Collique. Y con la concretización del mismo, esperan la adhesión de más asociados, el mismo que en un futuro se sumaran a su lucha emprendida.

El manejo de la dirección y presidencia, será una constante tensión entre la base norte y sur. Ya que por la separación geográfica, hace que de alguna manera sus problemas sean vistos de manera aislada. Por otro 
lado en la base de Lima Sur, crece una mayor concientización y ganas de querer emprender más acciones, se espera la pronto realización de proyectos de vivienda fuera de Lima metropolitana, en el distrito de cañetano de Asia.

\section{Relación con actores y agentes de su entorno}

Las relaciones que tejen cada integrante con otros actores y actoras son provechosas muchos de esos actores son dirigentes de AA. HH. Que buscan mejorar las condiciones de vida de sus allegados; los dirigentes son quienes contactan con organizaciones, $\mathrm{ONG}$, para facilitar los trámites para obtener por ejemplo luz, agua, muros de contención y, cuestiones de materia legal; claro que no direccionan la organización, pero aportan en algo a mejorarlo. Por otro lado, su relación con el mercado inmobiliario es casi inexistente, al mercado inmobiliario no le interesa que existan organizaciones sociales que brinden beneficios colectivos, sino es todo lo contrario, el mercado inmobiliario está más pendiente de la oferta ya demanda de tierras privadas y públicas.

La ONG CENCA ha sido de gran ayuda en materia legal pues ha contribuido en formular el proyecto de ley y la ordenanza municipal en la gestión actual del gobierno central y en la gestión de Susana Villarán, por supuesto ha contribuido a capacitar a los dirigentes, "pero somos nosotros los que tómanos la dirección política, enfatiza Rubén”; CENCA tiene le objetivo de acercarse más a la población, porque es como un vehículo que le ayuda a generar más confianza en esta organización.

El Frente Amplio (FA), esta organización política surge como un nuevo vínculo tejido, para continuar con las reivindicaciones sociales de la organización, según parece a largo plazo. El MST-MP apoyó la campaña política del (FA) con la condición de que sean apoyados desde el congreso con el proyecto de ley que se estancó en el gobierno que esta de salida.

Con la Municipalidad Metropolitana de Lima existe poco contacto, es casi nula la comunicación entre la municipalidad y la organización. Este solo dejo en medio camino la ordenanza municipal que elaboraron junto a CENCA, esto sin duda generó una caída en la organización entre conflictos y desconfianza entre los actores de la organización en mención.

Existe mucha indiferencia a esta organización como el Ministerio de Vivienda que no muestra signo de querer conversar con esta organización menos de ver cuáles son sus demandas. La Municipalidad de Comas no muestra interés ni una preocupación por estos pobladores que están en su jurisdicción y por su puesto por el Gobierno Central que no aplica políticas que estén direccionados para la población de bajos recursos como son los integrantes de esta organización es evidente el desinterés sobre todo del estado para tratar de remediar las consecuencia de las políticas neoliberales que ha desplazado a los que menos tienen.

\section{Pisando tierra, hacia la consolidación de una or- ganización social:}

El derecho a la vivienda es una de las primeras vías en todo un proceso de urbanización confuso y algo desordenado que se ha dado en Lima desde los años 60 con los abarrotamientos de los cerros y las zonas periféricas de la capital del país. Cabe mencionar que posterior a la iniciativa de adquirir una vivienda de carácter urgente surge la posibilidad de ser integrados a la ciudad en condición de ciudadanos, ser aceptados no solo por su fuerza de trabajo sino para establecer roles de participación ciudadana, esto a través, primero, del reconocimiento de sus viviendas, para luego poder establecerse dentro de la Lima pujante que hoy conocemos.

Ahora el devenir luego de adquirir una vivienda digna, y acceder a estos roles de ciudadanía son claros y objetivos, pues en ejemplos claros que existen y han venido siendo estudiados se nota una posterior forma de necesidad que sería clara de proponer como alternativa al MST-MP, pues los ciudadanos con sus viviendas dignas y reconocimiento social deberían de involucrarse por la integración completa al desarrollo de la ciudad como tal, construcción de migrantes des/ organizados que se establecieron en un momento histórico de nuestro país, y son los hijos de estos migrantes los que se ven en la necesidad no de repetir pero sí de establecer nuevos roles de vivienda y de ciudadanía, esto es a lo que aspira realmente toda una colectividad llena de olvidos y lagunas por parte del Estado, que como se mencionó en un principio de esta sección del artículo, es casi opresor y a la vez muy dejado por involucrar los beneficios de los más necesitados al rumbo o plan de gobierno que se plantea. El MST-MP quiere trascender mediante sus acciones y consolidarse como movimiento social, así como determinar la vivienda 
digna como una necesidad latente a nivel nacional. El aspecto social se ha manejado, solo falta que la atención del gobierno se dé de una forma satisfactoria, logre transmitir y reproducir estas experiencias en todo el territorio y a la población marginada o más necesitada. Las relaciones económico-sociales y solidarias que se han denotado en toda la extensión del artículo esperan seguir reproduciéndose y variando conforme se den nuevas experiencias y exista la suficiente fuerza para poder luchar, esto desde la medida de trabajar con las poblaciones y desde la potestad de trabajar con prudencia bajo lo estipulado como organización.

\section{Conclusiones}

No es difícil percibir que el derecho a la vivienda, el habitat o a la ciudad, viene siendo un fenómeno social aun no solucionado o tocado a fondo por las políticas sociales del Estado. Las políticas de desarrollo referidas al derecho a la vivienda, vienen siendo aún ineficaz, enmarcadas a la lógica del mercado y al servicio de grandes constructoras e inmobiliarias.

También como producto de ello hemos visto que el MST-MP ha resultado ser una nueva experiencia dentro de la basta historia de este fenómeno social basado de tomas de tierras. Las tomas de tierras en un contexto general, en la actualidad, han devenido también a relaciones referidas al tráfico de terrenos, alejados estos de relaciones de construcción y organización colectiva.

Ello tiene implicancias en el MST-MP, por una parte, porque ya ha habido confrontación con los traficantes de terreno, y por otro lado el tráfico de terreno va tomando notoriedad en distintos lugares de la sociedad. Por otro lado concluir que su relación con el estado podría tomar nuevos giros dentro del MSTMP, como lo dicho por uno de sus dirigentes: "Ahora tenemos 20 amigos en el congreso", ello podría incentivar nuevas negociaciones para procesos de formalización y servicios de primera necesidad en el MST-MP.

Ahora adentrándonos más al MST- MP, para esta organización es primordial conseguir un suelo para las familias o personas que no tienen lugar donde vivir, ello tiene un acercamiento a la economía social y solidaria, por el hecho de conseguirlo a través de la movilización y la organización colectiva con el objetivo de vivir bien o dignamente. Teniendo como bandera de lucha la consigna de "vivienda digna", en la realidad el MST-MP sigue otra dinámica, que es la de nego- ciar la compra de tierras privadas como es el caso de la experiencia asentada en Collique (Jesús de Nazareno). En Jesús de Nazareno se desenvuelven ciertas políticas de cooperación entre las familias, realizadas por medio faenas para luego dar píe a la construcción de sus viviendas, pero ello parte más como una obligación para permanecer dentro, ello ha ido corroyendo el mismo sentido de solidaridad, y muchos han optado por retirarse y buscar otro tipo de alternativas. Ello aun no logra calar como forma de identidad colectiva que los identifique de forma determinante como economía alternativa al capitalismo basado en la autogestión y reciprocidad.

Se deduce entonces que en este caso las relaciones de reciprocidad y de construcción colectiva de las viviendas estuvo presente con gran intensidad al inicio de la experiencia, cargados de fervor y movilización, luego esas relaciones se han ido desgastando tanto por conflictos internos como por la propia alienación presente siempre tanto en lugares donde el capitalismo ha llegado a desarrollarse y fortalecerse, como también donde ha venido a parar de forma estrepitosa. Así, cuando la organización es débil, las ideas de mercado, de consumo e individualistas, van cobrando forma y eclosionando mundos de la vida acorde a satisfacciones personales cargados de deseos superficiales sin un sentido más humano y solidario; la corriente es fuerte.

Un ejemplo claro de ello es la experiencia en Lomas de Corvina (Villa María del Triunfo) Donde una vez que el desarrollo humano va superando necesidades básicas la lógica va cambiando también, cada uno está en lo suyo, ya no hay muchas faenas, y las ganas de seguir ampliando y construyendo la organización va cambiando pro ganas de seguirse superando y satisfacer necesidades personales.

Esto producto de conflictos internos dentro de la organización, donde la consigna de lucha ha ido mutando a lógicas de clientelismo o de compra y venta de terreno, esto por una parte como una forma de facción, mientras la otra "facción" una lógica más de confrontación y de lucha directa contra los aparatos burocráticos y represivos para adquirir la tierra.

Ahora, a todo esto a que llamamos relaciones de economía social y solidaria ¿̇acaso siempre terminara por caer en lógicas individualistas y de consumo?, o es acaso que si la organización continua las relaciones de economía social y solidaria también pero de diferente forma.

La indagación ahora debe estar orientada a verificar el tipo de valor de uso y valor de cambio. El antes 
y el después de una ruptura (asumiendo que esto paso) con las lógicas del sistema, que pude suceder antes o después. Por un lado, el tipo de uso anterior, actual y futuro que le dan o quieren dar la tierra, en qué circunstancias se suscitan dichas rupturas y plantear preguntas como: ¿El tipo de uso debe ser constante o debe variar? ¿De qué forma influyen las consecuencias positivas y negativas de los cambios en el tipo de uso? Y el tipo de cambio, acaso el tipo de cambio debe solo ser guiada por la lógica de la ganancia acumulativa y personal o existe un tipo de ganancia las ligado a enriquecer las relaciones de solidaridad y reciprocidad y, como van constituyendo la representación simbólica del colectivo. Se hace necesario tener conocimiento de esto cuando se están constituyendo nuevas experiencias en Asia (Cañete) y en San Juan de Lurigancho.

\section{Bibliografía}

Arroyo, R. y Romero, A. (2008). "Lima Metropolitana y la globalización: plataforma de integración subordinada o espacio de autodeterminación en América Latina”, en: Córdova Montúfar, M. (coordinador) Lo urbano en su complejidad: una lectura desde América Latina, Quito: FLACSO Sede Ecuador, Ministerio de Cultura del Ecuador.

Burga, J. (2006). El ocaso de la barriada. Lima: Ministerio de Vivienda, Construcción y Saneamiento.

Calderón, J. (2006). Mercado de tierras urbanas, propiedad y pobreza. Lima: SINCO Editores.

Calderón, J. (2005). La ciudad ilegal. Lima: Lima en el siglo XX UNMSM.

Calderón, J. (2015). El derecho a la ciudad en América Latina y los enfoques sobre la ciudad informal. Revista de sociología №25, pg. 115-128.

García, Waldo (2013). El mítico poncho negro [Mensaje en un blog]. Recuperado de http://peruanoenasturias.blogspot.pe/2013/01/el-mitico-poncho-negro.html

Henry, E. (1978). Estado y movimiento de pobladores. Lima: Fondo Editorial, Pontificia Universidad Católica del Perú.

Raúl Guerrero de los Ríos, A. S. (1977). La trampa urbana: ideología y problemas urbanos, el caso de Lima. Lima: Desco, Centro de Estudios y Promoción del Desarrollo. 